\title{
Perspektiven
}

der Finanzierung

und Vergütung

der medizinischen

Rehabilitation

in Deutschland

Günter Neubauer

15.1 Einleitung - 283

15.2 Struktur der Versorgung, Finanzierung und Vergütung - 283

15.2.1 Die Angebotsseite - 284

15.2.2 Die Nachfrageseite - 285

15.2.3 Die Patientensteuerung - 286

15.3 Das Vergütungssystem in der medizinischen Rehabilitation - 287

15.3.1 Die Vertragspartner - 287

15.3.2 Systematik möglicher Vergütungssysteme - 288

15.3.3 Abrechnungseinheiten in der Rehabilitation - 288

15.3.4 Das Bewertungsverfahren - Findung der Entgelthöhe - 289

15.3.5 Zuschläge und Abschläge als komplementäre Vergütungskomponenten - 290 


\subsection{Aktuelle Reformdiskussion - 290}

15.4.1 Erweiterung der Finanzierungsquellen - 290

15.4.2 Differenzierte fallbezogene Vergütung in der Diskussion - 291

15.4.3 Komplexpauschalen ein Ziel? - 293

15.4.4 Digitalisierung der Rehabilitationsnachsorge - 294

15.5 Ausblick - 294

Literatur - 294 


\section{- Zusammenfassung}

Die medizinische Rehabilitation in Deutschland ist insgesamt gut aufgestellt, führt aber gleichwohl in der Gesundheitsversorgung ein Dasein als Aschenputtel. Sowohl die Angebotsseite wie die Nachfrageseite sind trotz entsprechender gesetzlicher Gebote im SGB IX wenig koordiniert. Vor allem die Finanzierungs- und Vergütungsmodalitäten sind immer noch historisch bestimmt. Insbesondere das Zusammenwirken der beiden großen Trägergruppen der Rehabilitation-der Krankenkassen und der regionalen Organisationen des Verbandes Deutscher Rentenversicherungsträger (VDR) - sollte intensiviert werden. Hinzu kommt als neue Aufgabe die Rehabilitation von Pflegebedürftigen. Für diese ist bislang keine adäquate Finanzierungform gefunden worden. Der Vorschlag, die Pflegeversicherung in die Finanzierung einzubinden, findet jedoch zunehmend Gehör.

Die Vergütung für Rehabilitationsleistungen erfolgt wenig differenziert nach normierten Behandlungstagen. Innerhalb einer Indikation gibt es so gut wie keine Differenzierung der Patientinnen und Patienten, obwohl Instrumente für eine Differenzierung nach Schweregrad durchaus verfügbar sind. Die Einführung einer fallbezogenen Vergütung auch in der Rehabilitation ist über Pilotprojekte nicht hinausgekommen. Ohne staatliches Eingreifen dürfte die Diskussion auch in der nächsten Zeit nicht vorankommen.

Medical rehabilitation in Germany is generally well positioned, but nevertheless leads an existence as a Cinderella in health care. Both the supply side and the demand side are poorly coordinated despite corresponding legal requirements in SGB IX. Especially the financing and remuneration modalities are still historically determined. In particular, the cooperation between the two major financiers of rehabilitation - the health insurance funds and the regional organisations of the Federal Association of German Pension Schemes (VDR) - should be intensified. In addition, the rehabilitation of those in need of long-term care is a new issue for which no adequate form of financing has been found so far. However, the proposal to involve long-term care insurance in the financing of rehabilitation is increasingly being heard.

The reimbursement for rehabilitation services takes place according to standardised treatment days. Within an indication, there is virtually no differentiation of patients, although instruments for grouping by severity are certainly available. The introduction of case-related remuneration in rehabilitation has not gone beyond pilot projects so far. Without government intervention, the discussion is unlikely to progress in the near future.

\subsection{Einleitung}

Gegenstand dieses Beitrags ist ausschließlich die medizinische Rehabilitation in Deutschland. Die berufliche und soziale Rehabilitation sind nicht Thema unserer Darlegungen.

Wir wollen in einem ersten Abschnitt das Rehabilitationssystem aus ökonomischer Perspektive darstellen. Insbesondere werden Angebot und Nachfrage als wichtige Bestimmungsgrößen für die Preisfindung in den Mittelpunkt gestellt. In einem zweiten Abschnitt soll dann das Vergütungssystem selbst einer Bewertung unterzogen werden. In einem dritten Abschnitt wollen wir auf die aktuelle Diskussion zur Weiterentwicklung der Finanzierung und Vergütung der medizinischen Rehabilitation eingehen. Den Abschluss bildet ein Ausblick auf die weitere Entwicklung in den nächsten Jahren, die insbesondere durch die Digitalisierung und den Einsatz von Robotern geprägt sein dürfte.

\subsection{Struktur der Versorgung, Finanzierung und Vergütung}

Um das komplexe System der Versorgung und Vergütung der Rehabilitation verständlich zu machen, bedienen wir uns vor allem der - Abb. 15.1. Dabei haben wir drei Strukturmerkmale in den Mittelpunkt gestellt: nämlich die Angebotsseite, die Nachfrageseite und schließlich die Koordination von Angebot und 


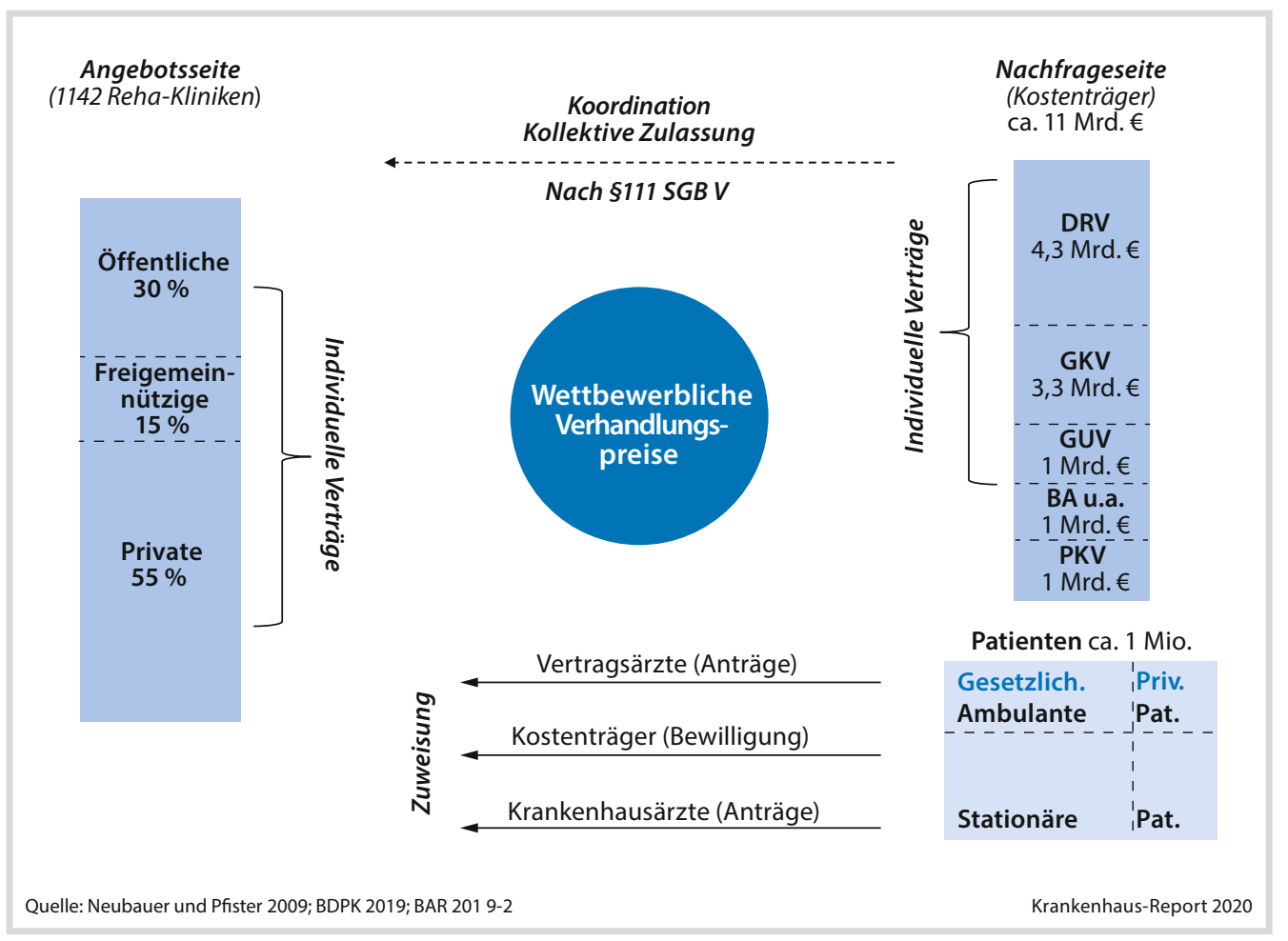

- Abb. 15.1 Struktur und Finanzierung der med. Reha (Quelle: Neubauer und Pfister 2009; BDPK 2019a; BAR 2019b)

Nachfrage unter anderem über die Vergütung (Neubauer und Pfister 2009).

\subsubsection{Die Angebotsseite}

Die Angebotsseite beschreibt die Rehabilitationskliniken als Leistungserbringer nach ihrer Trägerschaft. Die insgesamt 1.142 Kliniken werden von privaten Trägern dominiert; ihr Anteil beträgt rund $55 \%$ (vgl. • Abb. 15.1). Eine Besonderheit stellen die Kliniken in öffentlicher Trägerschaft der Deutschen Rentenversicherung (DRV) dar. Diese sind sowohl Anbieter als auch Nachfrager von Rehabilitationsleistungen. Das führt immer wieder zur Diskussion einer etwaigen Interessenkollision.

Ähnlich wie bei den Krankenhäusern ist auch bei den Rehabilitationskliniken eine zwei- fache Konzentration zu beobachten: Zum einen nehmen die Betriebsgrößen zu und zum anderen bilden sich Konzerne, die über eine Vielzahl von Betrieben verfügen. Bei den Betriebsgrößen schrumpfen die Kliniken mit weniger als 100 Betten und wachsen die Betriebe mit 150 Betten und mehr. Bei den Unternehmen wachsen die privaten Kettenbetriebe durch Übernahme von kleinen Unternehmen mit nur einen oder zwei Betrieben. Das größte Rehabilitationsunternehmen umfasst mittlerweile deutlich mehr als 100 Rehabilitationskliniken, die zum großen Teil von Dritten übernommen worden sind und einer Private-Equity-Gesellschaft gehören. Die Zahl der Klinikbetriebe insgesamt hingegen sinkt seit geraumer Zeit langsam (BDPK 2019a).

Das Leistungsspektrum selbst wird von der orthopädischen Rehabilitation dominiert. Die Indikation Kardiologie hingegen verliert lang- 
sam an Bedeutung, während die psychosomatische Indikation rasch zunimmt.

Neben der stationären Rehabilitation entwickelt sich derzeit eine ambulante Rehabilitation, die als wohnortnahe, familienfreundliche und kostengünstige Behandlungsvariante langsam an Marktanteilen gewinnt (BDPK 2019a).

Je nach der Zielsetzung der Rehabilitationsbehandlung wird zwischen einer postakuten Anschlussrehabilitation und präventivorientierten Heilverfahren unterschieden. Bei der Ersteren soll durch die Rehabilitation die Funktionsfähigkeit eines Patienten nach einem Krankenhausaufenthalt wieder soweit hergestellt werden, dass der Patient ohne fremde Hilfe zu Hause leben kann.

Ziel der Heilverfahren ist es, bei sozialversicherungspflichtigen Arbeitnehmern eine drohende Arbeitsunfähigkeit zu vermeiden oder zumindest deren Risiko zu verringern. Für die Heilverfahren gilt der Leitspruch „Reha vor Rente“.

\subsubsection{Die Nachfrageseite}

Die Nachfrageseite gliedert sich in drei Untergruppen, die jeweils unterschiedliche Funktionen der Nachfrage beinhalten, nämlich die Kostenübernahme bzw. Vergütung, die Leistungsinanspruchnahme und letztlich die Lenkung der Nachfrage bzw. Patienten.

\subsubsection{Die Kosten- bzw. Leistungsträger}

Bei den Kostenträgern, die sich auch häufig als Leistungsträger bezeichnen, dominieren die Deutsche Rentenversicherung (DRV) und die gesetzlichen Krankenkassen (GKV). Beide finanzieren etwa $70 \%$ des Leistungsvolumens. Einen deutlich geringeren Marktanteil hat die gesetzliche Unfallversicherung (GUV). Weitere Träger der medizinischen Rehabilitation sind die Bundesanstalt für Arbeit, die Jugendhilfe, die Sozialhilfe sowie Träger der Kriegsopferversorgung ( $\$ 6$ SGB IX). Hinzu kommt die private Krankenversicherung (PKV) als Rehabilitationsträger (vgl. • Abb. 15.1).

Die Leistungen, die der medizinischen Rehabilitation zugeordnet werden, sind in den $\mathrm{Pa}$ ragraphen 1 bis 5 des SGB IX beschrieben. Insbesondere werden Leistungen zur Erlangung bzw. Sicherstellung der Teilhabe von Behinderten betont - ein Leistungsbereich, der in der medizinischen Rehabilitation als bislang vernachlässigt bezeichnet werden muss.

Für die verschiedenen Rehabilitationsträger, die durch die rund 110 Krankenkassen und 16 regionalen Rentenversicherungsträger an Vielfalt gewinnen bzw. an Transparenz verlieren, gibt es keine einheitlichen Vergütungsregeln.

Allerdings zeichnet sich eine Konzentration der Rehabilitationsträger dahingehend ab, dass die kleinen Krankenkassen sich zu Vertragsgemeinschaften zusammenschließen und die regionalen Rentenversicherungsträger der Deutschen Rentenversicherung sich ebenfalls bei Preisverhandlungen abstimmen. So haben sich Teile der Betriebskrankenkassen zu der GWQ ServicePlus AG zusammengeschlossen.

Bei der Anerkennung und Zulassung der einzelnen Rehakliniken als Vertragspartner und damit verbunden dem Recht auf Vergütung für die Behandlung von entsprechend versicherten Patienten haben die Krankenkassen wie auch die Deutsche Rentenversicherung jeweils einheitliche Kriterien entwickelt. Im Mittelpunkt steht die Strukturqualität in Form der personellen und sachlichen Ausstattung. Elemente der Prozessqualität werden insbesondere von der Deutschen Rentenversicherung vermehrt gefordert, etwa in Form eines indikationsspezifischen Behandlungsplans (BAR 2019a). Die Zulassung als Vertragspartner der GKV beinhaltet nicht automatisch auch die Zulassung durch die DRV. Eine Zulassung bedeutet jedoch keinen Anspruch auf Belegung. 


\subsubsection{Die Leistungsinanspruch- nahme - Rolle der Patienten}

Entsprechend den dominierenden Rehabilitationsträgern, DRV und GKV, lassen sich zwei große Gruppen von Patientinnen und Patienten unterscheiden: zum einen Erwerbstätige, deren Erwerbsfähigkeit durch Krankheit gefährdet ist, zum anderen bereits Erkrankte, also Patienten, die in ärztlicher Behandlung sind oder deren akutmedizinische Behandlung beendet ist und die eine anschließende Ausheilphase bzw. gesundheitsstärkende Maßnahme benötigen. Die klassische Kur als gesundheitsstärkende Maßnahme, ohne dass die oben beschriebenen Risiken bzw. Zustände gegeben sind, wird nur noch im Bereich von "Mutter-und-Kind-Kuren" durch die GKV gewährt.

Ist der Anspruch auf eine Rehabilitationsmaßnahme gegeben, so hat der Anspruchsberechtigte gemäß $\$ 9$ SGB IX ein Wahlrecht hinsichtlich der jeweiligen Rehabilitationsklinik. Berechtigte Wünsche des Rehabilitanden dürfen von den Kostenträgern nicht abgelehnt werden. Dieses Wahlrecht behindert vertragliche Preisvereinbarungen zwischen Rehabilitationskliniken und Kostenträgern bislang wenig, da von dem Wahlrecht kaum Gebrauch gemacht wird.

Wenig Steuerungswirkung entfaltet auch die vorgesehene Kostenbeteiligung der Patienten an ihrem Rehabilitationsaufenthalt. Grundsätzlich muss der Patient $10,00 €$ pro Tag entrichten, was nicht als Kostenbeteiligung, sondern als Vorteilsausgleich für die nicht notwendige häusliche Versorgung eingestuft wird.

An Bedeutung haben die akut-stationären Patienten gewonnen, was durch das fallbezogene DRG-Entgeltsystem unterstützt wird (BDPK 2019a). Die postakute Ausheilphase wird verstärkt in die Anschlussrehabilitation verlagert. Diese enge Verknüpfung der Leistungen hat auch den Ruf nach sogenannten Komplexpauschalen ausgelöst (vgl. auch $>$ Abschn. 15.4.3).

Privatversicherte erhalten je nach Versicherungsvertrag Rehabilitationsleistungen. Die
Leistungen der DRV stehen diesem Personenkreis ebenfalls zur Verfügung, soweit sie Mitglied in der gesetzlichen Rentenversicherung sind.

Generell werden die Rehabilitanden älter - insbesondere im Leistungsbereich der GKV ist dies heute schon spürbar, da die Akutmedizin immer älter werdende Patienten auch schwierigen Operationen unterzieht und diese frühzeitig entlässt. Im Bereich der gesetzlichen Rentenversicherung (GRV) ist mit der schrittweisen Erhöhung des Renteneintrittsalters ein ähnlicher Trend zu spüren. Mittelfristig ist somit eine wachsende Nachfrage bzw. ein wachsender Bedarf an Rehabilitationsleistungen insbesondere in der Indikation Geriatrie - zu erwarten (Lewis 2009; Heuvel 2017).

\subsubsection{Die Patientensteuerung}

Die Patientensteuerung entspricht aus ökonomischer Sicht einer Nachfragesteuerung. Diese erfolgt über mehrere Akteure und auf mehreren Wegen.

Rehabilitationsleistungen erfordern stets eine ärztliche Bedarfsfeststellung. Daher sind die behandelnden Ärzte auch gleichzeitig eine initiale Steuerungsinstanz. Sie bestimmen, welche Patienten aus medizinischer Sicht eine Rehabilitationsmaßnahme erhalten sollten. Neben den niedergelassenen Vertragsärzten können auch Krankenhausärzte den Bedarf einer postakuten Rehabilitation feststellen.

Welche Rehabilitationsklinik die Ärzte empfehlen, hängt u.a. davon ab, inwieweit die Rehabilitationsklinik sich mit den verordnenden Ärzten, insbesondere den Krankenhausärzten, abgestimmt hat. Und da die Krankenhäuser heute zu einem gezielten Entlassmanagement verpflichtet sind, bevorzugen die Krankenhäuser Rehabilitationskliniken, die mit ihnen vertragliche Vereinbarungen getroffen haben. Inhalte solcher Vereinbarungen sind vor allem die Abstimmung der nachfolgenden Therapie sowie die Kommunikation und Dokumentation. Häufig übernimmt in 
den Krankenhäusern das Entlassmanagement die Auswahl der geeigneten Rehabilitationsklinik, während die Ärzte lediglich den Bedarf einer Rehabilitation feststellen (Köhler und Wiederer 2017).

Das letzte Wort, ob und welche Art von Rehabilitation gewährt wird, haben freilich die Rehabilitationsträger. Diese prüfen neben der Bedarfsnotwendigkeit vor allem die Wirtschaftlichkeit der ärztlichen Verordnungen. Schließlich müssen sie die Kosten der Rehabilitation übernehmen. Die Bewilligungsquoten der verschiedenen Rehabilitationsträger liegen zwischen 60 und $90 \%$ der beantragten Rehabilitationsmaßnahmen. Für das Jahr 2017 liegt die Ablehnungsquote der GKV für die Anschlussrehabilitation bei $8 \%$ und für Heilverfahren der DRV bei $38 \%$. Die letztere Zahl halbierte sich allerdings aufgrund von erfolgreichen Widerspruchsverfahren (BDPK 2019a). Im Referentenentwurf zum Reha- und Intensivpflege-Stärkungsgesetz (RISG 2019) wird die Ablehnung einer Rehabilitationsmaßnahme durch die Krankenkassen erschwert.

Mit der Bewilligung gibt der Kostenträger gleichzeitig die jeweilige Rehabilitationsklinik an, in der die Rehabilitation durchgeführt werden soll. Damit steuern die Rehabilitationsträger die Patienten in solche Rehabilitationskliniken, mit denen sie spezielle Verträge über Preise und Leistungen abgeschlossen haben. Je gezielter die Rehabilitationsträger ihre Versicherten steuern können, umso eher sind die Rehabilitationskliniken bereit, sich auf entsprechende Preis-Mengen-Konditionen vertraglich festzulegen.

An dieser Stelle tauchen zwei Probleme auf: Zum einen haben die Patienten wie erwähnt ein Wahlrecht hinsichtlich der Kliniken, was zum Konflikt mit den vertraglichen Vereinbarungen ihrer Versicherung führen kann. Zum zweiten ist die DRV versucht, ihre Versicherten bevorzugt in ihre eigenen Kliniken zu steuern. Dies gilt insbesondere, wenn die Preise der eigenen Kliniken höher liegen als die vergleichbarer privater Einrichtungen. Das Problem einer Be- nachteiligung der Privaten im Wettbewerb wird dadurch entschärft, dass die DRV dezidierte Anforderungen an die Qualität der Rehabilitationskliniken stellt, die eine höhere Vergütung rechtfertigen. Die Krankenkassen haben die eigenen Rehakliniken weitgehend abgegeben und sind insofern freier in der Steuerung ihrer Patienten. Im RISG 2019 ist vorgesehen, dass die Wahlmöglichkeiten der Patienten dadurch gestärkt werden, dass sie bei einer Abweichung von der Empfehlung der Kostenträger nur noch die Hälfte der Mehrkosten zu übernehmen haben.

\subsection{Das Vergütungssystem in der medizinischen Rehabilitation}

\subsubsection{Die Vertragspartner}

Die Zulassung einer Rehabilitationsklinik für die Behandlung der Versicherten eines Rehabilitationsträgers begründet zwar den Anspruch auf Vergütung, doch ist die Höhe der Vergütung Verhandlungsgegenstand. Jeder rechtlich selbständige Rehabilitationsträger kann mit jeder zugelassenen Rehabilitationsklinik einen eigenen Vergütungsvertrag abschließen. Tatsächlich schließen sich vor allem die kleinen Krankenkassen zu Vertragsgemeinschaft zusammen, um auf diese Weise mehr Marktmacht zu erlangen, aber auch um den Verwaltungsaufwand zu optimieren.

Im Bereich der Rentenversicherung übernimmt die jeweils wichtigste regionale Rentenversicherung die Verhandlungsführerschaft. Die Ergebnisse werden dann von allen belegenden Rentenversicherungen übernommen. Ähnliches gilt für die Unfallversicherung (Oberscheven 2013).

Die Kliniken sehen sich somit einer Reihe verschiedener Kostenträger gegenüber, die jeweils eigene Vertragsabschlüsse verknüpft mit eigenen Leistungsvorgaben anstreben. In der - Abb. 15.1 haben wir diese Struktur transparent gemacht und mit Zahlen hinterlegt. 


\subsubsection{Systematik möglicher Vergütungssysteme}

Vergütungssysteme können generell mit drei Kriterien systematisiert werden. Das erste Kriterium ist die Vergütungs- oder Abrechnungseinheit. Es wird damit die Frage „Was wird abgerechnet?" beantwortet. Die Abrechnungseinheiten sollten möglichst gut die gewünschten Leistungseinheiten beschreiben, damit eine leistungsbezogene Vergütung erreicht wird. Im Gesundheitsbereich werden viele verschiedene Abrechnungseinheiten praktiziert. Das Spektrum reicht von der Abrechnung von Einzelleistungen bis hin zu einer Fallpauschale.

In - Abb. 15.2 haben wir die Systematik mit einigen wichtigen Elementen angereichert. Eine Spezifizierung für die Rehabilitation nehmen wir im anschließenden Abschnitt vor.

Neben den Abrechnungseinheiten in der ersten Säule stehen in der zweiten Säule die Bewertungsmethoden. Hier unterscheiden wir zwei Gruppen: nämlich die Bewertung nach Kosten und die Bewertung mit Preisen. Die kostenorientierte Bewertung kann wiederum nach individuellen Istkosten, nach durchschnittlichen Istkosten oder nach Sollkosten erfolgen.

Die Preise können entweder durch Verhandlungen oder über Märkte ermittelt werden. In der Praxis gibt es häufig auch - je nach Marktlage - Mischlösungen.

In der dritten Säule, die wir auch als Ergänzungsmodul bezeichnen, erfolgen Korrekturen zu den ersten beiden Modulen. Ergänzungen können sowohl zu den Abrechnungseinheiten wie auch zur Bewertung hinzukommen. So könnte eine telematische Nachbetreuung von Patienten neben einer Pauschale abgerechnet werden.

\subsubsection{Abrechnungseinheiten in der Rehabilitation}

In der medizinischen Rehabilitation hat sich in Deutschland, aber auch im Europa der Behandlungstag als Abrechnungseinheit eingebürgert. Dabei wird der Abrechnungstag in der Regel nach der jeweiligen Behandlungsindikation wie z.B. Orthopädie oder Psychosomatik differenziert. Allerdings können die Behandlungstage nicht unbegrenzt abgerechnet werden, denn ihre Zahl ist pro Patient in der Regel pauschal begrenzt. Überschreitungen müssen durch die Klinik gesondert beantragt und durch den jeweiligen Rehabilitationsträger zusätzlich genehmigt werden. Fälschlicherweise wird hier öfters auch von Fallpauschalen gesprochen.

Der Behandlungstag als Abrechnungseinheit hat wenig Bezug zu der erbrachten Leistung und damit auch zu den entstandenen Kosten. Vor allem der Schweregrad einer erforderlichen Behandlung wird so gut wie gar nicht berücksichtigt. Eine gewisse Berücksichtigung des Behandlungsaufwandes findet sich in der Differenzierung nach Indikation. Eine tiefer gehende Differenzierung wird in der Neurologischen Rehabilitation vorgenommen. Dort werden vier Phasen der Rehabilitation unterschieden, je nach zeitlichem Abstand zur Akutbehandlung und damit auch indirekt nach dem Schweregrad. Dies ist jedoch die Ausnahme in Deutschland. In der Schweiz ist das System der Leistungsdifferenzierung in der Neurologischen Rehabilitation viel stärker nach Schweregrad verfeinert (Ranneberg 2006).

Der differenzierte Behandlungsfall wird in der Rehabilitation bislang kaum verwendet, auch wenn die Diskussion um eine Angleichung der Vergütung an das DRG-Entgeltsystem der Krankenhäuser immer wieder geführt wird. Bereits vor etwa 20 Jahren gab es mehrere Modellversuche zur fallbezogenen Vergütung in der Rehabilitation (Neubauer und Nowy 2000). Die Ergebnisse waren durchaus positiv, gleichwohl waren weder Krankenkassen noch Rehabilitationskliniken bereit, die Mo- 


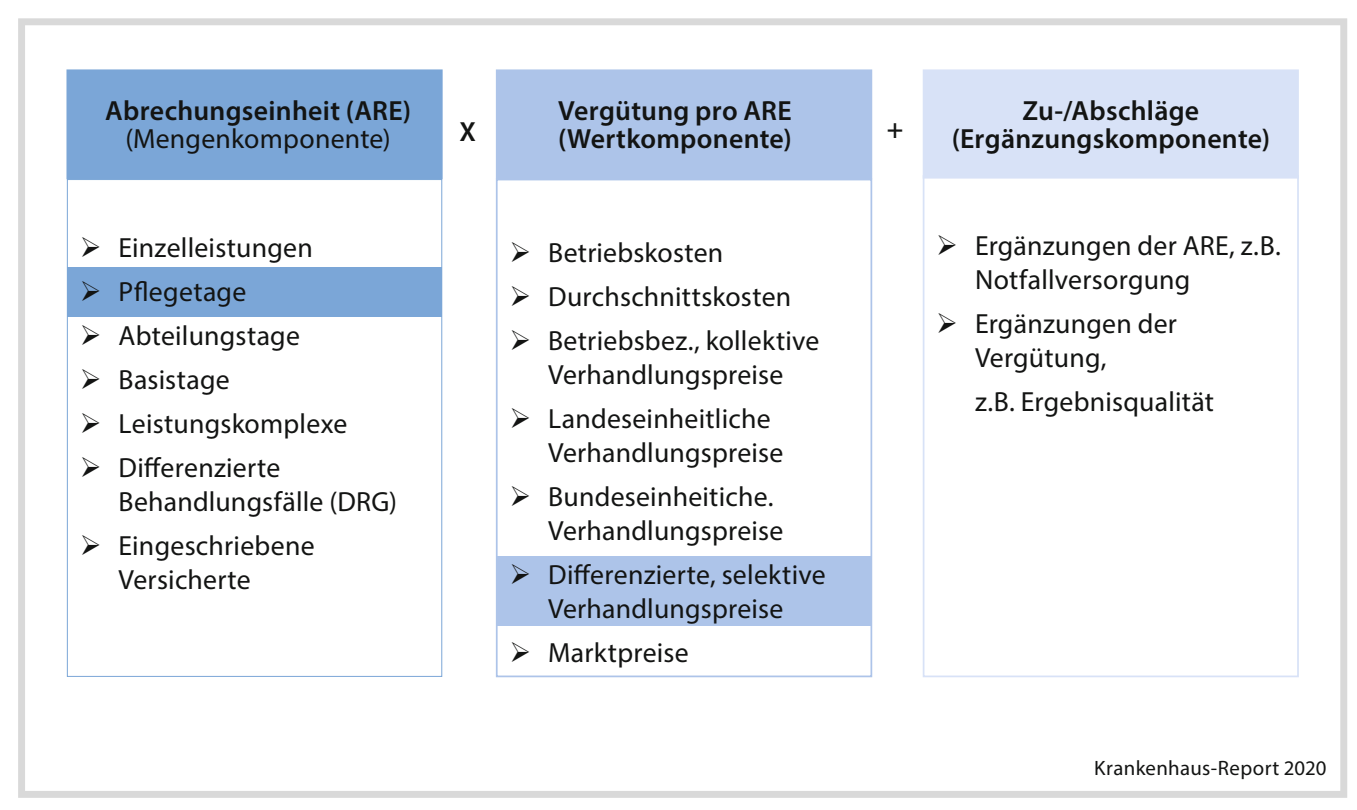

- Abb. 15.2 Basismodule eines leistungsbezogenen Vergütungssystems (Quelle: Neubauer 2019; eigene Darstellung)

dellversuche in die Regelversorgung umzusetzen. Mit den Gründen beschäftigen wir uns in > Abschn. 15.4.3 ausführlich.

\subsubsection{Das Bewertungsverfahren - Findung der Entgelthöhe}

In der deutschen Rehabilitation dominiert das Prinzip der Verhandlungspreise. Wie wir schon oben im $>$ Abschn. 15.2 bei der Beschreibung des Marktes für Rehabilitationsleistungen betont haben, verhandeln die großen Rehabilitationsträger mit den einzelnen Kliniken oder auch mit den großen Klinikunternehmen die indikationsspezifischen Preise. Verhandelt werden so genannte Vollkostenpreise, d. h. die Investitionskosten werden über die Preise finanziert, ohne extra berücksichtigt zu werden.

Im Bereich der GKV haben die großen Krankenkassen einen beträchtlichen Marktanteil, den sie noch dadurch erhöhen, dass sie ihre Patienten auf wenige Vertragskliniken konzentrieren. Dadurch geraten diese Kliniken unter einen erheblichen Preisdruck. Nach Berechnungen des BDPK (2019a) liegen die Entgelte der Krankenkassen deutlich unter dem kostendeckenden Preis. Doch da andererseits der Auslastungsgrad der Kapazitäten, gemessen an der Belegung der aufgestellten Betten, über die ökonomische Ertragslage entscheidet, sind die Kliniken bereit, Preisnachlässe zu verhandeln, sofern dafür ihr Belegungsgrad entsprechend ansteigt (BAR 2019a).

Seit dem Jahr 2011 können die Verhandlungspartner bei Nicht-Einigung eine Schiedsstelle anrufen. Diese Möglichkeit wird vonseiten der Kliniken zurückhaltend bis gar nicht genutzt, da mit einer Vergütungsvereinbarung keine Belegungsgarantie verbunden ist. Die Kliniken befürchten, dass sie bei einer durch Schiedsspruch festgesetzten Vergütung bei der Belegung entsprechend benachteiligt werden (Gommermann 2011).

Trotz der vielfältigen, unterschiedlichen Verhandlungspreise gibt es eine gewisse Preistransparenz innerhalb der GKV, da sich die Krankenkassen in begrenztem Umfang austauschen. 
In der DRV ist die Vielfalt der indikationsspezifischen Preise deutlich geringer. Zwar haben die regionalen Rentenversicherungsträger Verhandlungsautonomie mit den Rehabilitationskliniken, doch erfolgt eine Abstimmung pro Klinik. Insbesondere bei den Preisverhandlungen mit den eigenen Kliniken stimmen sich die verschiedenen regionalen Rentenversicherungen ab, sodass alle den gleichen Preis mit einer DRV-Rehabilitationsklinik vereinbaren. Generell vergüten die Rentenversicherungsträger nach Auskunft verschiedener Klinikmanager die Leistungen höher als die Krankenkassen. Die Begründung dafür ist, dass die DRV höhere Anforderungen an die Dokumentation und Qualität der Rehabilitationskliniken stellt, was diese in erster Linie als zusätzlichen bürokratischen Aufwand einschätzen.

\subsubsection{Zuschläge und Abschläge als komplementäre Vergütungskomponenten}

Entsprechend dem Verhandlungsprinzip werden für Sonderleistungen Zuschläge gezahlt, wenn diese zwischen den Vertragspartnern vereinbart wurden. Zwar haben GKV und DRV jeweils für ihren Bereich eigene Vorgaben für die Struktur- und teilweise auch die Prozessqualität, deren Erfüllung Voraussetzung für die $\mathrm{Zu}$ lassung als Vertragspartner ist (Spiegel 2019).

Zuschläge erhalten Rehabilitationskliniken, wenn sie eine besondere Gruppe von Patientinnen und Patienten therapieren, etwa Übergewichtige oder Dialysepatienten. Ebenso kann für eine telematische Nachbetreuung von Patienten ein Entgeltzuschlag vereinbart werden.

Für Ergebnisqualität - gemessen an der Rückkehrquote der Patienten an den Arbeitsplatz - werden in der DRV Vergütungsaufschläge verhandelt. Die DRV hat darüber hinaus eine eigene Rehabilitationsform mit der Bezeichnung „Berufsorientierte Rehabilitation (BOR)“ entwickelt.

Generell ist das System der Zuschläge bzw. Abschläge sowie der Definition, welche Leis- tungen zusätzlich abgerechnet werden können, wenig transparent. Hervorzuheben ist, dass kaum Abschläge, aber sehr wohl Zuschläge im Vergütungssystem zur Anwendung kommen.

\subsection{Aktuelle Reformdiskussion}

Die aktuelle Reformdiskussion konzentriert sich vor allem auf zwei Themen: Zum einen wird die Einbeziehung der Pflegekassen in die Finanzierung gefordert, um den Grundsatz Reha vor Pflege systemgerecht umsetzen zu können (Zerth et al. 2019). Ein zweiter Diskussionsbereich kreist um die Abrechnungseinheit Behandlungsfall analog zu den DRGs (Bublitz 2019). Schließlich gibt es eine anhaltende Diskussion um den Beitrag der Rehabilitation zur Kosteneinsparung in der Gesundheitsversorgung bzw. zur Steigerung der Produktivität der Beschäftigten (Bublitz 2019). Letztere Diskussion wollen wir hier nur am Rande anführen, da sie nicht direkt unserem Thema zuzuordnen ist.

\subsubsection{Erweiterung der Finanzierungsquellen}

Infolge der Diskussion zur Versorgung von Pflegebedürftigen wird derzeit auch eine verstärkte Rehabilitation für Pflegebedürftige gefordert. So soll der medizinische Dienst der Krankenkassen bei der Feststellung der Pflegebedürftigkeit auch gleichzeitig den Bedarf für eine medizinische Rehabilitation feststellen (Breuninger 2017).

Die Rehabilitationsmaßnahmen werden aber von den Krankenkassen finanziert und nicht von den begünstigten Pflegekassen. Da die Krankenkassen mit ihren Beiträgen im Wettbewerb stehen, befürchten die Rehabilitationskliniken, dass Rehabilitationsmaßnahmen für Pflegebedürftige weiterhin eher restriktiv bewilligt und durchgeführt werden. Die Forderung, nun auch vonseiten des Verbandes der 
privaten Kliniken (BDPK) vorgetragen, lautet, dass die Pflegekassen die Finanzierung der Rehabilitation für Pflegebedürftige übernehmen, weil sie dadurch Ausgaben einsparen können (Zerth et al. 2019). Die Krankenkassen wiederum halten dagegen, dass die Pflegekassen nicht über die notwendige Expertise verfügen, um effizient Vergütungsverhandlungen mit Rehabilitationskliniken durchführen zu können.

Ein Kompromissvorschlag dazu läuft darauf hinaus, dass zwar weiterhin die Krankenkassen die Verhandlungen mit den Rehabilitationskliniken führen, ähnlich wie bisher aber die Pflegekassen die Kosten direkt übernehmen (BDPK 2019b). Und da die Pflegekassen nicht über den Beitragssatz im Wettbewerb stehen, unterstellt man, dass die Rehabilitation nachdrücklicher unterstützt wird. Schließlich profitieren die Pflegekassen direkt davon, dass die Pflegebedürftigkeit ihrer Versicherten vermieden bzw. reduziert wird, während die Krankenkassen nur die Auswirkungen der finanziellen Belastung auf ihren Beitragssatz spüren, mit dem sie im Wettbewerb stehen. Mit Blick auf die steigende Zahl Pflegebedürftiger sollte dieser Ansatz weiterverfolgt werden.

In jedem Fall bedarf eine entsprechende Änderung der Finanzierung der politischen Unterstützung in der Form, dass entsprechende Regelungen in das SGB V übernommen werden. Hierzu gibt es bislang (2019) keine parlamentarische Initiative. Ein weiteres Manko der Finanzierung ist, dass im Morbi-RSA keine eigene Pauschale für Rehabilitationspatienten vorgesehen ist. Dies führt bei den Krankenkassen, da sie im Beitragswettbewerb stehen, zu einem unverhältnismäßigen Spardruck in Bezug auf die Rehabilitation.

Für die DRV wird ein jährliches Ausgabenvolumen vorgegeben, das sich nur wenig am tatsächlichen Bedarf orientiert. Zwar wurde die strikte Budgetierung aufgelockert, indem ein Demografiefaktor in das Budget bis 2022 eingebaut wurde, doch kann von einer bedarfsgerechten Versorgung nicht gesprochen werden.

\subsubsection{Differenzierte fallbezogene Vergütung in der Diskussion}

Mit der Einführung des DRG-Entgeltsystems für die Krankenhäuser wurden entsprechende Überlegungen auch im Hinblick auf die medizinische Rehabilitation weltweit (Fischer et al. 2006), aber auch in Deutschland diskutiert (Neubauer und Nowy 2002).

In einer Reihe von Pilotprojekten wurden Gemeinsamkeiten und Besonderheiten der medizinischen Rehabilitation zur DRGKrankenhausvergütung diskutiert und erprobt. An den Pilotprojekten nahmen verschiedene Krankenkassen und auch verschiedene Klinikbetreiber teil (Neubauer und Ranneberg 2005). Die Rentenversicherungsträger verhielten und verhalten sich zurückhaltend bis skeptisch (Beyerle 2019).

Hindernisse für eine freiwillige Einführung einer fallbezogenen Vergütung für die medizinische Rehabilitation in Deutschland, häufig in Anlehnung an die DRGs auch als Rehabilitations-Behandlungsgruppen (RBG) bezeichnet (Neubauer und Pfister 2009), waren vor allem die folgenden:

(1) Begrenzte Eignung des ICD-Schlüssels für die Gruppierung der Patienten aus Sicht der Rehabilitationsmediziner:

Der ICD-Schlüssel wurde von Rehabilitationsmedizinern durch den ICF (International Classification of Functioning, Disability and Health)-Schlüssel ersetzt (Ranneberg 2006). Dieser bildet über vier verschiedene Kategorien den Gesundheitszustand von Rehabilitationspatienten differenziert ab. Doch mangelte es diesem Schlüssel an praktischer Handhabbarkeit. Dieser Schlüssel ist als Basis für eine fallbezogene Vergütung auf absehbare Zeit nicht anwendbar und wird in den deutschen Rehabilitationskliniken auch nicht verwendet. Für eine praktisch anwendbare Fallgruppierung wurden bei der Bildung von Rehabilitationsbehandlungsgruppen (RBGen) lediglich vier Kriterien eingesetzt, die alle bereits heute in Rehakliniken 


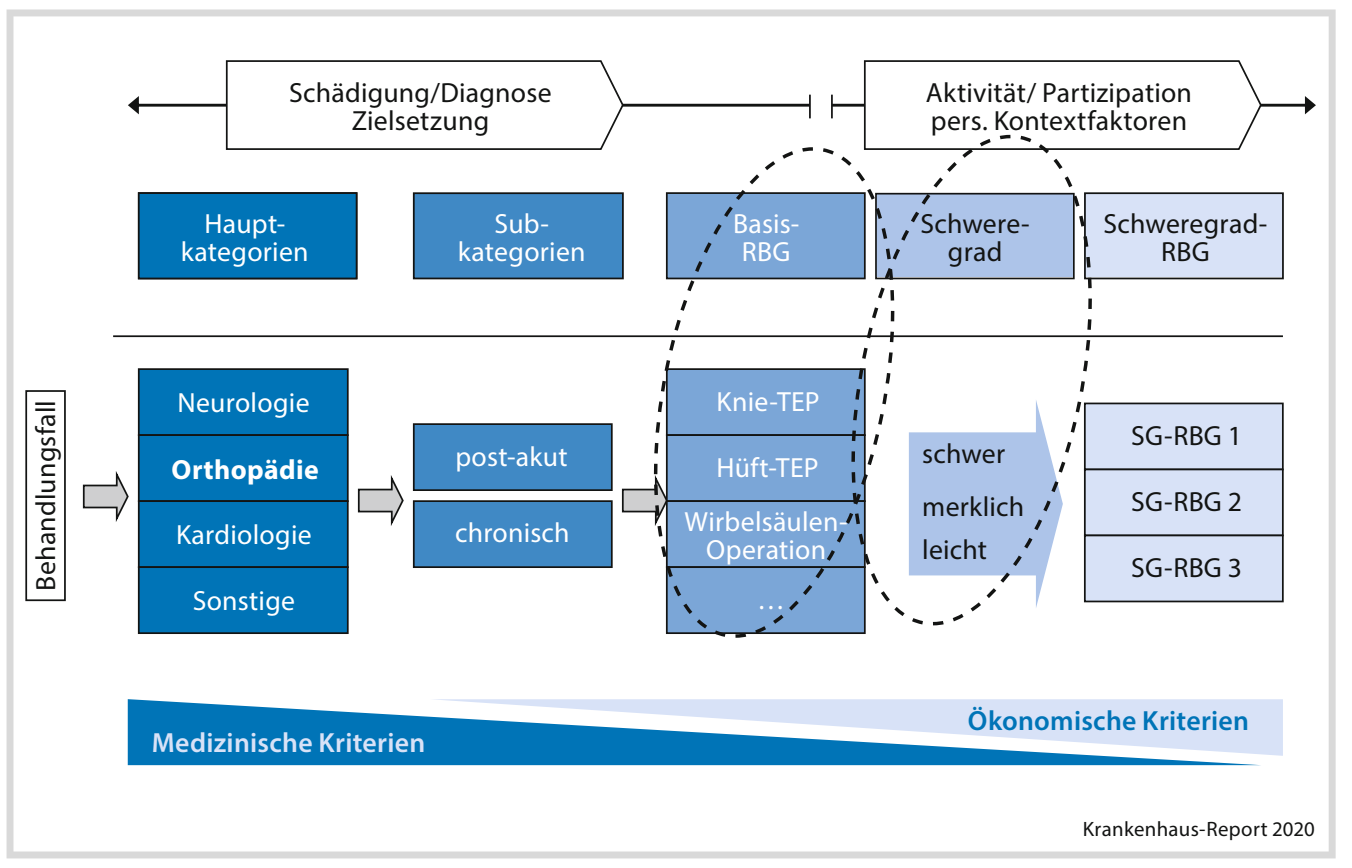

- Abb. 15.3 Das RBG-Klassifikationsmodell (Quelle: Neubauer 2007)

verwendet werden (Neubauer und Pfister 2009). - Abb. 15.3 verdeutlicht diesen $\mathrm{Zu}$ sammenhang.

(2) Keine Berücksichtigung des spezifischen Behandlungsaufwandes in der Rehabilitation aus Sicht der Kliniken:

Um kostengleiche Fallgruppen zu bilden, genügt als Merkmal die jeweilige Indikation in Kombination mit dem ICD-Schlüssel bzw. den wichtigsten Behandlungsgebieten wie z. B. Schulter, Knie oder Wirbelsäule, um in Kliniken entsprechende Fallkalkulationen durchzuführen.

(3) Fehlende Berücksichtigung des Schweregrades der Behandlung aus Sicht der Rehabilitationsmediziner:

Ähnlich wie im DRG-System lassen sich auch in der Rehabilitation für die einzelnen Patientengruppen differenzierende Schweregrade ermitteln, die als Kostengewichte dann in die Vergütung eingehen können. Im System der RBGen wurden als Schweregradmerkmale die Einstufung der Patienten nach dem Barthel-Index bzw. FIM-In- dex herangezogen (Neubauer 2011). Beide Indices messen die Funktionsfähigkeit der Patienten, wobei der FIM-Index psychosoziale Einschränkungen mit einbezieht, während der Barthel-Index auf die körperlichen Fähigkeiten abstellt.

(4) Hoher zusätzlicher Dokumentationsaufwand aus Sicht der Kliniken:

Als Begründung für die Ablehnung von Fallpauschalen führen die Rehabilitationsunternehmen, aber auch die Krankenkassen insbesondere die Erfahrungen der Krankenhäuser mit der Einführung der DRG-Abrechnung an. Als abschreckende Beispiele werden die hohen Anforderungen an das interne Rechnungswesen und die komplexe Abrechnung im DRG-Entgeltsystem genannt. Die aktuelle Diskussion zum Umbau des DRG-Entgeltsystems unterstützt diese Einschätzung.

(5) Bei kostenneutraler Umstellung vermissen die Krankenkassen finanzielle Vorteile: Eine Umstellung des Vergütungssystems darf keine der beteiligten Seiten - weder die 
Kostenträger noch die Rehabilitationskliniken - von vornherein benachteiligen bzw. begünstigen, sonst wird die Umstellung von der jeweiligen Seite blockiert. Doch müssen auch die einzelnen Rehabilitationskliniken wie auch die einzelnen Kostenträger möglichst ausgabenneutral gehalten werden, um zu erwartende Widerstände auszuräumen. Dies erfordert zwar einige Rechenarbeit, die das InEK sicherlich übernehmen könnte. In einer Anpassungsphase sollten Übergangsregelungen gelten, wie es auch bei der Einführung der DRGs gehandhabt wurde.

Weil aber eine aufwandsneutrale Umstellung für keine Seite rasche Vorteile verspricht, will auch keine der Seiten eine entsprechende Entwicklung vorantreiben. Dem Argument, dass eine schweregradbasierte Fallgruppierung bei zunehmend älteren Patienten automatisch den Rehabilitationskliniken höhere Erlöse bringt, ohne dass die Krankenkassen über Verhandlungen zustimmen müssen, wird nicht getraut.

Im Ergebnis lässt sich für Deutschland und auch global feststellen, dass bislang von den betroffenen Parteien noch keine fallbezogene Vergütung freiwillig vereinbart worden ist (Kimberly und Pouvourville 1993; Fischer et al. 2006). In allen Ländern, in denen DRG-basierte Vergütungssysteme eingeführt worden sind, ist der Staat aktiv geworden. Auch für Deutschland gilt diese Prämisse, tatsächlich läuft aber derzeit (2019) in Deutschland die politische Meinungsbildung in die entgegengesetzte Richtung.

\subsubsection{Komplexpauschalen ein Ziel?}

In der Diskussion werden häufig auch Komplexpauschalen in der Form diskutiert, dass die Vergütung von Krankenhaus und postakuter Rehabilitation über eine Pauschale vergütet wird (Neubauer 2005).
Als Vorteil einer solchen Komplexpauschale wird die flexible, frühzeitige Verlegung von Patientinnen und Patienten in die Rehabilitationsklinik gesehen. Insbesondere werden dadurch schonendere Operationsmethoden und eine nachfolgende rasche Mobilisierung der $\mathrm{Pa}$ tienten durch die Rehabilitation unterstützt. Auf der anderen Seite können Rehabilitationskliniken ihren Behandlungsablauf auf den entsprechenden Bedarf der Patienten abstimmen. Eine verkürzte Verweildauer im Krankenhaus kann durch eine frühzeitige, bedarfsadäquate Aufnahme von Patienten in die Rehabilitationsklinik kompensiert werden (Neubauer und Minartz 2007). Freilich bedingt das, dass die Rehabilitationsklinik Patienten aufnimmt, die noch nicht voll rehabilitationsfähig sind in dem Sinne, dass sie sich schon selbstständig bewegen können.

Und hier setzt auch der Widerstand gegen eine Komplexpauschale an: Rehabilitationskliniken sind nur bereit, Akutpatienten, die noch nicht rehabilitationsfähig sind, aufzunehmen, wenn sie für den erhöhten Aufwand von den Krankenhäusern entschädigt werden. Dies lehnen die Krankenhäuser jedoch in der Regel ab, insbesondere wenn sie einen anderen Träger haben. Gehören hingegen Krankenhaus und Rehabilitationsklinik einem Eigentümer, findet der finanzielle Interessenausgleich im Unternehmen statt. Das Unternehmen kalkuliert dann den Nettoeffekt.

Einen besonderen Widerstand gegen Komplexpauschalen artikuliert die DRV: Sie lehnt es generell $a b$, dass Rehabilitationskliniken nicht rehabilitationsfähige Patienten aufnehmen. Der Grund hierfür ist, dass die Krankenkassen hiervon Vorteile hätten, da sie für die Krankenhausvergütung zuständig sind, während die Rentenversicherer die Nachteile in Form eines höheren Rehabilitationsaufwandes übernehmen müssten. Von daher wurden Komplexpauschalen bislang nur im Geltungsbereich der Krankenkassen erprobt und auch teilweise vereinbart (Köhler und Wiederer 2017).

Als Fazit gilt: Generell werden Komplexpauschalen nur unter finanziellen Aspekten be- 
wertet. Die Vorteile einer koordinierten, durchgängigen Behandlung von Patienten sowie einer bedarfsgerechten Verlegung vom Krankenhaus in die Rehabilitationsklinik werden nicht gesehen bzw. unterschätzt. Insbesondere die zunehmende Zahl an geriatrischen Patienten kann durch eine frühzeitige Mobilisierung in der Rehaklinik gesundheitlich profitieren. $\mathrm{Ob}$ das gesetzlich vorgeschrieben Entlassmanagement hier eine Lösung bringt, bleibt abzuwarten.

\subsubsection{Digitalisierung der Rehabilitationsnachsorge}

Die Digitalisierung hat die Rehabilitation bisher nur begrenzt erreicht. Ein Grund hierfür ist die schwache Investitionskraft der Rehabilitationskliniken. Vielversprechend sind erste Ansätze zur digital organisierten Nachbetreuung von Rehabilitationspatienten. So setzt die Klinikgruppe REHASAN das digitale Nachsorgeprogramm „Rehasonanz" mit Unterstützung von Krankenkassen durchaus erfolgreich ein (Gesser et al. 2014). Generell haftet der Rehabilitation das Problem an, dass Patienten danach sehr schnell wieder in alte Verhaltensweisen zurückfallen und der Rehabilitationseffekt verhallt. Hier setzen Nachbetreuungsprogramme an, die mithilfe digitaler Medien die Patienten auch zu Hause weiter betreuen.

Von der Möglichkeit, Apps zu verordnen, wie es im Digitalen Versorgungsgesetz (BMG 2019) vorgesehen ist, könnte auch die Rehabilitationsnachsorge profitieren. Auf diesem Weg kann den Patienten eine omnipräsente Anleitung angeboten werden, mit deren Hilfe sie die positiven Effekte der Rehabilitation im Alltag möglichst lange erhalten können.

\subsection{Ausblick}

In die Zukunft gerichtet kann man davon ausgehen, dass die älter werdende Bevölkerung, ebenso wie die älter werdenden Erwerbstäti- gen $\mathrm{zu}$ einer medizinischen wie wirtschaftlichen Höherbewertung der Rehabilitation führen werden (BDPK 2019c). Eine Berücksichtigung dieser wertschöpfenden Beiträge ist in der Vergütung bislang jedoch nicht vorgesehen.

Auch der Fachkräftemangel zeigt Auswirkungen auf die Rehabilitationskliniken. Insbesondere das Pflegestärkungsgesetz benachteiligt die Rehakliniken im Vergleich zu den Krankenhäusern. Zu befürchten ist, dass Pflegekräfte aus der Rehabilitation in die Krankenhäuser abwandern, da dort die Vergütung höher ist.

Die Digitalisierung hat die Rehabilitationskliniken bisher nur teilweise - im Bereich der Organisation - erreicht. Wenig bis gar nicht wird die Digitalisierung im Bereich der Therapie genutzt, obwohl gerade hier eine Reihe von digitalen Assistenzsystemen bis hin zu Robotern von der medizintechnischen Industrie angeboten werden.

Derzeit besteht eine Investitionsschwäche der Rehabilitationskliniken, die zumindest teilweise durch die Form der Vergütung mit verursacht wird. Wenn die Rehabilitation ihre wichtige Funktion in der Gesundheitsversorgung wahrnehmen soll, ist eine Neuorientierung in den nächsten Jahren im Sinne einer stärkeren Ergebnisorientierung der Vergütung und einer bedarfsorientierten Finanzierung erforderlich.

\section{Literatur}

BAR (2019a) Spitzenverbände der RehaLeistungserbringer. Gemeinsame Positionen zur zukünftigen Sicherung und Weiterentwicklung der Rehabilitation und Teilhabe für die laufende 19. Legislaturperiode. https://www. bar-frankfurt.de/fileadmin/dateiliste/1-News-

Seiten/1-Reha_und_Teilhabe/downloads/2019_

Positionspapier_Spitzenverb\%C3\%A4nde_der_

Reha-Leistungserbringer.pdf. Zugegriffen: 22. Juli 2019

BAR (2019b) Reha-Info 1/2019

BDPK (2019a) fact sheet. BDPK, Berlin

BDPK (2019b) Änderungsbedarf SGB V - medizinische Reha

BDPK (2019c) Forderungen des BDPK zur medizinischen Rehabilitation der Gesetzlichen Krankenversicherung 
Beyerle B (2019) Fallbezogene Vergütung für die Rehabilitation. Vortrag auf dem 3. Nationalen Reha-Forum, Berlin, 22. März 2019

Bundesministerium für Gesundheit (BMG) (2019) Ärzte sollen Apps verschreiben können. Gesetz für eine bessere Versorgung durch Digitalisierung und Innovation (Digitale-Versorgung-Gesetz - DVG). Berlin. https://www.bundesgesundheitsministerium.de/ digitale-versorgung-gesetz.html. Zugegriffen: 11. November 2019

Breuninger K (2017) Rehabilitation vor und bei Pflege. KU Gesundheitsmanagement 9:50-52

Bublitz T (2019) Reha im Focus. KU Gesundheitsmanagement 3:46-48

Fischer W, Blanco J, Mäder M, Zangger P, Conti FM, Bapst L, Huwiler B (2006) Das TAR-System und andere Patientenklassifikationssysteme für die Rehabilitation. TAR-Forschungsbericht und Kurzbeschrieb von Systemen aus Deutschland, Frankreich, Australien und den USA. Zentrum für Informatik und wirtschaftliche Medizin (ZIM), Wolfertswil

Gesser U, Schwartz F, Roschewsky F (2014) "rehasonanz.de" - Wirkung eines ärztlich betreuten OnlinePortals. Welt Krankenversicherung 10:232-235

Gommermann R (2011) Schiedsstellen in der Rehabilitation und was das Infektionsschutzgesetz für die Rehabilitation sonst noch bedeutet. Verband der Ersatzkassen e. V. (vdek). Landesvertretung Thüringen, Vortrag, Bad Berka

van den Heuvel D (2017) Ist die Zukunft der Rehabilitation geriatrisch? KU Gesundheitsmanagement 9:6062

Kimberly JR, de Pouvourville G (1993) The migration of managerial innovation: diagnosis-related groups and health care administration in western Europe. Jossey-Bass, San Francisco

Köhler M, Wiederer R (2017) Gesundheit im Jahr 2030. KU Gesundheitsmanagement 9:57-59

Lewis P (2009) Gegenwärtige und zukünftige Herausforderungen an die medizinische Rehabilitation: eine Analyse unter Berücksichtigung von demographischem Wandel, medizinisch-technischem Fortschritt sowie Lebensstilveränderungen. Peter Lang, Frankfurt

Neubauer G (2005) Versorgungssteuerung über Vergütungsanreize. In: Klauber J, Robra BP, Schellschmidt H (Hrsg) Krankenhaus-Report 2005. Schattauer, Stuttgart, S 37-54

Neubauer G (2007) Wann kommen die Reha-DRG? Vortrag auf dem 6. Nationalen DRG-Forum, Berlin, 20. Apr. 2007
Neubauer G (2011) Steigenden Behandlungsbedarf in der Rehabilitation über Schweregradgruppen abbilden und vergüten. In: Neubauer G (Hrsg) Ein Jahrzehnt Gesundheitsökonomie und Gesundheitspolitik 2000-2010. Institut für Gesundheitsökonomik (IfG), München, S 145-152

Neubauer G (2019) Ökonomisierung der medizinischen Rehabilitationsversorgung.Vortrag an der Akademie für Gesundheit und Lebensmittelsicherheit. München

Neubauer G, Minartz C (2007) Komplexpauschalen für die Akut- und Rehabilitationsbehandlung in der Herzchirurgie. Abschlussbericht für das Jahr 2007. Institut für Gesundheitsökonomik (IfG), München

Neubauer G, Nowy R (2000) Gesundheitsökonomische Evaluationen. In: Gerdes N, Jäckel WH, Weidemann $\mathrm{H}$ (Hrsg) Protos-II: Evaluation der Einführung von Fallpauschalen in den kardiologischen und orthopädischen Rehabilitationskliniken der Wittgensteiner Kliniken Allianz. Steinkopff, Darmstadt, S 36-42

Neubauer G, Nowy R (2002) Das DRG-System erfordert Fallpauschalen in der Rehabilitation. f\&w 2:179-181

Neubauer G, Pfister F (2009) Die stationäre medizinische Rehabilitation: Struktur, Finanzierung und Trends. Betriebliche Prävention 9:418-422

Neubauer G, Ranneberg J (2005) Entwicklung von Rehabilitationsbehandlungsgruppen (RBG) für die Kardiologie und Orthopädie - Ergebnisse eines Forschungsprojektes. Rehabilitation 44:34-43

Oberscheven M (2013) Neuausrichtung der stationären Heilverfahren. Dguv Forum 1-2:60-63

Ranneberg J (2006) Ansätze zur Patientenklassifikation in der medizinischen Rehabilitation aus gesundheitsökonomischer Perspektive. Peter Lang, Frankfurt

RISG (Referentenentwurf der Bundesregierung) (2019) Entwurf eines Gesetzes zur Stärkung von Rehabilitation und intensivpflegerischer Versorgung in der gesetzlichen Krankenversicherung (Rehaund Intensivpflege-Stärkungsgesetz - RISG). https://www.bundesgesundheitsministerium.de/ fileadmin/Dateien/3_Downloads/Gesetze_und_ Verordnungen/GuV/R/Referentenentwurf_RISG. pdf. Zugegriffen: 19. Aug. 2019

Spiegel M (2019) Die Bedeutung von Qualitätsmanagement in Rehabilitations-Einrichtungen aus Sicht von Qualitätsmanagement-Beauftragten. Gesundheitsökonomie Qual 24:141-146

Zerth J, Wasem J, Matusiewicz D (2019) „Reha vor Pflege“ - zur Problematik einer undeutlichen gesundheitspolitischen Regelstrategie - ein Positionspapier. Gesundheitsökonomie Qual 24:121-125 
Open Access Dieses Kapitel wird unter der Creative Commons Namensnennung 4.0 International Lizenz (http:// creativecommons.org/licenses/by/4.0/deed.de) veröffentlicht, welche die Nutzung, Vervielfältigung, Bearbeitung, Verbreitung und Wiedergabe in jeglichem Medium und Format erlaubt, sofern Sie den/die ursprünglichen Autor(en) und die Quelle ordnungsgemäß nennen, einen Link zur Creative Commons Lizenz beifügen und angeben, ob Änderungen vorgenommen wurden.

Die in diesem Kapitel enthaltenen Bilder und sonstiges Drittmaterial unterliegen ebenfalls der genannten Creative Commons Lizenz, sofern sich aus der Abbildungslegende nichts anderes ergibt. Sofern das betreffende Material nicht unter der genannten Creative Commons Lizenz steht und die betreffende Handlung nicht nach gesetzlichen Vorschriften erlaubt ist, ist für die oben aufgeführten Weiterverwendungen des Materials die Einwilligung des jeweiligen Rechteinhabers einzuholen. 